\title{
A finite difference method for heat equation in the unbounded domain
}

\author{
Quan Zheng ${ }^{\text {a }}$ and Xin Zhao \\ College of Science, North China University of Technology, Beijing, 100144, China
}

\begin{abstract}
The numerical method of the one-dimensional non-homogeneous heat equation on an unbounded domain is considered. Two exact artificial boundary conditions are applied on two artificial boundaries to limit the original problem onto a bounded computational domain. Then the finite difference method is developed by using the method of the reduction of order for the control equation and artificial boundary conditions. It is proved that the finite difference scheme is stable and convergent with the order 2 in space and order 3/2 in time under an energy norm. A non-homogeneous numerical example demonstrates the unconditional stability and the accuracy of the algorithm.
\end{abstract}

Keywords: non-homogeneous heat equation; unbounded domain; artificial boundary condition; finite difference method; error estimate.

\section{Introduction}

Heat equation arises from many fields, for examples, the heat transfer, fluid dynamics, astrophysics, finance or other areas of applied mathematics. For the problem on unbounded domains, Han and Huang (2002) in [1] and [2] derived an exact artificial boundary condition, obtained the corresponding numerical solution for heat equations by FEM and FDM in one-dimensional and two-dimensional cases, showed the effectiveness with numerical examples, but had no error estimate. Wu and Sun (2004) in [3] applied the artificial boundary condition in [1] to investigate the numerical solution of heat equation on the semi-infinite interval, proving the unconditional stability and convergence with order 2 in space and the order $3 / 2$ in time under an energy norm.

In this paper, we consider the following Cauchy problem of one-dimensional non-homogeneous heat equation:

$$
\begin{gathered}
u_{t}-\sigma u_{x x}=f(x, t),-\infty<x<+\infty, 0<t \leq T, \\
u(x, 0)=\phi(x), \quad-\infty<x<+\infty, \\
u(x, t) \rightarrow 0 \text {, when }|x| \rightarrow+\infty, 0 \leq t \leq T .
\end{gathered}
$$

\footnotetext{
a Corresponding author : zhengq@ncut.edu.com
} 
where $\sigma$ is diffusion coefficient determining heat conduction rate, density and heat capacity of the material. The initial term $\phi(x)$ and the source term $f(x, t)$ have compact supports in space.

Introducing artificial boundaries

$$
\Gamma_{l}=\left\{(x, t) \mid x=x_{l}, 0 \leq t \leq T\right\} \text { and } \Gamma_{r}=\left\{(x, t) \mid x=x_{r}, 0 \leq t \leq T\right\}
$$

such that $\operatorname{supp}\{f(x, t)\} \subset\left[x_{l}, x_{r}\right] \times[0, T]$ and $\operatorname{supp}\{\phi(x)\} \subset\left[x_{l}, x_{r}\right]$. According to the work of Han and Huang in [1] and Wu and Sun in [3], we can reduce the initial problem to an initial-boundary problem on a bounded domain:

$$
\begin{gathered}
\frac{\partial u}{\partial t}-\sigma \frac{\partial v}{\partial x}=f(x, t), x_{l}<x<x_{r}, 0<t \leq T, \\
v-\frac{\partial u}{\partial x}=0, x_{l}<x<x_{r}, \\
u(x, 0)=\phi(x), x_{l}<x<x_{r}, \\
v\left(x_{l}, t\right)=\frac{1}{\sqrt{\pi \sigma}} \int_{0}^{t} \frac{\partial u\left(x_{l}, \lambda\right)}{\partial \lambda} \frac{1}{\sqrt{t-\lambda}} d \lambda, \quad 0<t \leq T, \\
v\left(x_{r}, t\right)=-\frac{1}{\sqrt{\pi \sigma}} \int_{0}^{t} \frac{\partial u\left(x_{r}, \lambda\right)}{\partial \lambda} \frac{1}{\sqrt{t-\lambda}} d \lambda, 0<t \leq T .
\end{gathered}
$$

\section{The finite difference scheme and corresponding error estimation}

We discretize the above problem as follows. Divide the domain $\left[x_{l}, x_{r}\right] \times[0, T]$ into an $M \times N$ mesh with the spatial size $h=\left(x_{r}-x_{l}\right) / M$ in $x$ direction and the time step size $\tau=T / N$ in $t$ direction respectively, where $M$ and $N$ are positive integers. Grid points $\left(x_{i}, t_{n}\right)$ are defined by $x_{i}=x_{l}+i h$ for $0 \leq i \leq M$ and $t_{n}=n \tau$ for $0 \leq n \leq N$. Moreover, denote $U_{i}^{n}$ be the numerical solution of $u(x, t)$ at $\left(x_{i}, t_{n}\right)$ and introduce the notations:

$$
\begin{aligned}
& U_{i-\frac{1}{2}}^{n}=\frac{1}{2}\left(U_{i}^{n}+U_{i-1}^{n}\right), U_{i}^{n-\frac{1}{2}}=\frac{1}{2}\left(U_{i}^{n}+U_{i}^{n-1}\right), \quad \delta_{x} U_{i-\frac{1}{2}}^{n}=\frac{1}{h}\left(U_{i}^{n}-U_{i-1}^{n}\right), \\
& \delta_{x}^{2} U_{i}^{n}=\frac{1}{h^{2}}\left(U_{i+1}^{n}-2 U_{i}^{n}+U_{i-1}^{n}\right), \quad \delta_{t} U_{i}^{n-\frac{1}{2}}=\frac{1}{\tau}\left(U_{i}^{n}-U_{i}^{n-1}\right), \\
& f_{i-\frac{1}{2}}^{n}=\frac{1}{2}\left(f_{i}^{n}+f_{i-1}^{n}\right), f_{i}^{n-\frac{1}{2}}=\frac{1}{2}\left(f_{i}^{n}+f_{i}^{n-1}\right), \quad\left\|U^{n}\right\|_{E}=\sqrt{h \sum_{i=1}^{M}\left(U_{i-\frac{1}{2}}^{n}\right)^{2}} .
\end{aligned}
$$

Define the grid function: $u_{i}^{n}=u\left(x_{i}, t_{n}\right), \quad 0 \leq i \leq M, n \geq 0$. The notations of $v$ are similar. Applying finite difference method for (4)-(8), we have 


$$
\begin{aligned}
& \delta_{t} u_{i-\frac{1}{2}}^{n-\frac{1}{2}}-\sigma \delta_{x} v_{i-\frac{1}{2}}^{n-\frac{1}{2}}-f_{i-\frac{1}{2}}^{n-\frac{1}{2}}=r_{1, i-\frac{1}{2}}^{n-\frac{1}{2}}, \quad 1 \leq i \leq M, n \geq 1, \\
& v_{i-\frac{1}{2}}^{n-\frac{1}{2}}-\delta_{x}{ }_{i-\frac{1}{2}}^{n-\frac{1}{2}}=r_{2, i-\frac{1}{2}}^{n-\frac{1}{2}}, \quad 1 \leq i \leq M, n \geq 1, \\
& \delta_{t} u_{i-\frac{1}{2}}^{n-\frac{1}{2}}-\sigma \delta_{x} v_{i-\frac{1}{2}}^{n-\frac{1}{2}}-f_{i-\frac{1}{2}}^{n-\frac{1}{2}}=r_{1, i-\frac{1}{2}}^{n-\frac{1}{2}}, \quad 1 \leq i \leq M, n \geq 1, \\
& { }_{i-\frac{1}{2}}^{n-\frac{1}{2}}-\delta_{x} u_{i-\frac{1}{2}}^{n-\frac{1}{2}}=r_{2, i-\frac{1}{2}}^{n-\frac{1}{2}}, \quad 1 \leq i \leq M, n \geq 1, \\
& u_{i}^{0}=\phi\left(x_{i}\right),
\end{aligned}
$$

where $\left|r_{1, i-\frac{1}{2}}^{n-\frac{1}{2}}\right| \leq c_{1}\left(\tau^{2}+h^{2}\right),\left|r_{2, i-\frac{1}{2}}^{n-\frac{1}{2}}\right| \leq c_{1} h^{2},\left|s_{1}^{n-\frac{1}{2}}\right| \leq c_{2} \tau^{\frac{3}{2}},\left|s_{2}^{n-\frac{1}{2}}\right| \leq c_{2} \tau^{\frac{3}{2}}, 1 \leq i \leq M, n \geq 1$, and $c_{1}$ and $c_{2}$ are constants independent of $\tau$ and h.

Omitting the small error terms in (8)-(13), we construct a finite difference scheme:

$$
\begin{gathered}
\delta_{t} U_{i-\frac{1}{2}}^{n-\frac{1}{2}}-\sigma \delta_{X} V_{i-\frac{1}{2}}^{n-\frac{1}{2}}-f_{i-\frac{1}{2}}^{n-\frac{1}{2}}=0, \quad 1 \leq i \leq M, n \geq 1 \\
V_{i-\frac{1}{2}}^{n-\frac{1}{2}}-\delta_{X} U_{i-\frac{1}{2}}^{n-\frac{1}{2}}=0, \quad 1 \leq i \leq M, n \geq 1, \\
V_{0}^{n-\frac{1}{2}}=\frac{2}{\sqrt{\pi \sigma}}\left[a_{0} U_{0}^{n-\frac{1}{2}}-\sum_{k=1}^{n-1}\left(a_{n-k-1}-a_{n-k}\right) U_{0}^{k-\frac{1}{2}}\right], n \geq 1 \\
V_{M}-\frac{1}{2}=-\frac{2}{\sqrt{\pi \sigma}}\left[a_{0} U_{M}^{n-\frac{1}{2}}-\sum_{k=1}^{n-1}\left(a_{n-k-1}-a_{n-k}\right) U_{M}^{k-\frac{1}{2}}\right], n \geq 1, \\
U_{i}^{0}=\phi\left(x_{i}\right), 0 \leq i \leq M .
\end{gathered}
$$

Theorem 1. The finite difference scheme (14)-(18) is equivalent to the following scheme (19)-(23) without intermediate variable:

$$
\begin{gathered}
\frac{1}{2}\left(\delta_{t} U_{i-\frac{1}{2}}^{n-\frac{1}{2}}+\delta_{t} U_{i+\frac{1}{2}}^{n-\frac{1}{2}}\right)-\sigma \delta_{x}^{2} U_{i}^{n-\frac{1}{2}}=\frac{1}{2}\left(f_{i-\frac{1}{2}}^{n-\frac{1}{2}}+f_{i+\frac{1}{2}}^{n-\frac{1}{2}}\right), \\
\delta_{t} U_{\frac{1}{2}}^{n-\frac{1}{2}}+\frac{2 \sigma}{h}\left[\frac{2}{\sqrt{\pi \sigma}}\left(a_{0} U_{0}^{n-\frac{1}{2}}-\sum_{k=1}^{n-1}\left(a_{n-k-1}-a_{n-k}\right) U_{0}^{k-\frac{1}{2}}\right)-\delta_{x} U_{\frac{1}{2}}^{n-\frac{1}{2}}\right]=f_{\frac{1}{2}}^{n-\frac{1}{2}}, \\
\delta_{t} U_{M-\frac{1}{2}}^{n-\frac{1}{2}}+\frac{2 \sigma}{h}\left[\frac{2}{\sqrt{\pi \sigma}}\left(a_{0} U_{M}^{n-\frac{1}{2}}-\sum_{k=1}^{n-1}\left(a_{n-k-1}-a_{n-k}\right) U_{M}^{k-\frac{1}{2}}\right)+\delta_{x} U_{M-\frac{1}{2}}^{n-\frac{1}{2}}\right]=f_{M-\frac{1}{2}}^{n-\frac{1}{2}},
\end{gathered}
$$




$$
\begin{gathered}
U_{i}^{0}=\phi\left(x_{i}\right), \\
\text { where } a_{m}=\frac{1}{\sqrt{\tau}}(\sqrt{m+1}-\sqrt{m}), m=1,2,3, \mathrm{~L} .
\end{gathered}
$$

We can obtain the stability and the convergence of the finite difference scheme using Gronwall inequality as follows by using the methods in [4] and [5].

Theorem 2. Let $\max _{x_{i} \leq x \leq x_{r}, 0 \leq t \leq T}|f(x, t)| \leq c_{3}, c_{3}$ be a positive constant. $\left\{U_{i}^{n}\right\}$ be the

Solution of the difference scheme $(14)-(18), u(x, t)$ be the solution of problem (4)-(8). Then

$$
\begin{gathered}
\left\|U^{n}\right\|_{E}^{2} \leq \frac{1}{1-\tau} \exp \left(\frac{2 \tau}{1-\tau}\right)\left(\left\|U^{0}\right\|_{E}^{2}+c_{3}^{2} T L\right), n=1,2, \mathrm{~L}, T / \tau, \\
\left\|u^{n}-U^{n}\right\|_{E}^{2} \leq \exp \left(\frac{2 T}{2-\tau}\right) \frac{2 c^{2} T}{2-\tau}\left[\sqrt{\frac{\sigma T}{\pi}}+(\sigma+2) L\right]\left(\tau^{\frac{3}{2}}+h^{2}\right)^{2}, \quad \mathrm{n}=1,2, \mathrm{~L},[\mathrm{~T} / \tau],
\end{gathered}
$$

where $c=\max \left\{c_{1}, c_{2}, c_{3}\right\}$.

\section{Numerical example}

We introduce the following definitions:

$$
\begin{aligned}
& L^{\infty} \text {-error: } E_{h, \tau}^{\infty}=\max _{1 \leq i \leq M}\left|U_{i}^{n}-u_{i}^{n}\right|_{h, \tau}, \quad L^{\infty} \text {-order: } \log _{2}\left(E_{h, \tau}^{\infty} / E_{h / 2}^{\infty}, \tau\right), \\
& L^{2} \text {-error: } E_{h, \tau}^{2}=\sqrt{h \sum_{i=1}^{M}\left[U_{i}^{n}-u_{i}^{n}\right]_{h, \tau}^{2}}, \quad L^{2} \text {-order: } \log _{2}\left(E_{h, \tau}^{2} / E_{h / 2}^{2}, \tau\right),
\end{aligned}
$$

\begin{tabular}{|c|c|c|c|c|c|c|c|c|c|}
\hline \multirow[b]{2}{*}{$\mathrm{M}$} & \multicolumn{4}{|c|}{$\mathrm{T}=1$} & \multicolumn{5}{|c|}{$\mathrm{T}=2$} \\
\hline & $\mathrm{N}$ & $L^{\infty}$-error & order & $L^{2}$-error order & $\mathrm{N}$ & $L^{\infty}$-error & order & $L^{2}$-error & order \\
\hline 56 & 10 & $4.7676 \mathrm{e}-3$ & ----- & $\begin{array}{ll}4.6564 e-3 \quad---- \\
\end{array}$ & 20 & $2.2550 \mathrm{e}-3$ & $\begin{array}{ll}---- \\
\end{array}$ & $2.1077 \mathrm{e}-3$ & $\begin{array}{ll}---- \\
\end{array}$ \\
\hline 95 & 20 & $1.6199 \mathrm{e}-3$ & 1.5574 & $1.6091 \mathrm{e}-31.5330$ & 40 & $7.5236 \mathrm{e}-4$ & 1.5836 & $7.1586 \mathrm{e}-3$ & 1.5579 \\
\hline 159 & 40 & $5.7385 \mathrm{e}-4$ & 1.4972 & $5.7163 \mathrm{e}-41.4931$ & 80 & $2.6194 \mathrm{e}-4$ & 1.5221 & $2.5128 \mathrm{e}-4$ & 1.5104 \\
\hline 267 & 80 & $2.0198 \mathrm{e}-4$ & 1.5065 & $2.0202 \mathrm{e}-4 \quad 1.5006$ & 160 & $9.1078 \mathrm{e}-5$ & 1.5241 & $8.8160 \mathrm{e}-5$ & 1.5111 \\
\hline
\end{tabular}

Example 1.Consider the following nonhomogeneous heat equation:

$$
\begin{gathered}
u_{t}-\sigma u_{x x}=\left(2 \sigma-1-4 x^{2}\right) e^{-x^{2}-t}, \quad-\infty<x<+\infty, 0<t \leq T, \\
u(x, 0)=e^{-x^{2}}, \quad-\infty<x<+\infty \\
u(x, t) \rightarrow 0 \text {, when }|x| \rightarrow+\infty, 0 \leq t \leq T .
\end{gathered}
$$

which has the exact solution $u(x, t)=e^{-x^{2}-t}$. The support of $\phi(x)$ and $f(x, t)$ is compact on the computational domain $\left[x_{l}, x_{r}\right]=[-5,5]$.

Table 1. Convergence w.r.t. $\tau$ of Example 1 for $\sigma=1$ and $h=\tau^{3 / 4}$. 
Table 2. Convergence w.r.t. h of Example 1 for $\sigma=1$ and $\tau=h^{4 / 3}$.

\begin{tabular}{|c|c|c|c|c|c|c|c|c|c|}
\hline \multicolumn{5}{|c|}{$\mathrm{T}=1$} & \multicolumn{5}{|c|}{$\mathrm{T}=2$} \\
\hline M & $\mathrm{N}$ & $L^{\infty}$-error & order & $L^{2}$-error order & $\mathrm{N}$ & $L^{\infty}$-error & order & $L^{2}$-error & order \\
\hline 25 & 7 & $1.9906 \mathrm{e}-2$ & ----- & 2.2418e-2 ----- & 14 & $9.0971 \mathrm{e}-3$ & ----- & $9.7143 \mathrm{e}-3$ & ----- \\
\hline 50 & 17 & $5.6867 \mathrm{e}-3$ & 1.8075 & $5.7087 \mathrm{e}-3 \quad 1.9734$ & 34 & $2.5422 \mathrm{e}-3$ & 1.8393 & $2.4764 \mathrm{e}-3$ & 1.8710 \\
\hline 100 & 42 & $1.4219 \mathrm{e}-3$ & 1.9998 & $1.4316 \mathrm{e}-3 \quad 1.9955$ & 84 & $6.3418 \mathrm{e}-4$ & 2.0093 & $6.1983 \mathrm{e}-4$ & 1.9719 \\
\hline 200 & 107 & $3.5441 \mathrm{e}-4$ & 2.0043 & $3.5782 \mathrm{e}-4 \quad 2.0003$ & 21 & $1.5651 \mathrm{e}-4$ & 2.0125 & $1.5461 \mathrm{e}-4$ & 2.0032 \\
\hline
\end{tabular}

We can see in Table 1 that the order of convergence agrees with the theoretical order $3 / 2$ in time during the different time periods, since Theorem 2 gives $O\left(\tau^{3 / 2}+h^{2}\right)=O\left(\tau^{3 / 2}\right)$ for $h=\tau^{3 / 4}$. Table2 illustrates the theoretical order 2 of convergence since Theorem 2 gives $O\left(\tau^{3 / 2}+h^{2}\right)=O\left(h^{2}\right)$ for $\tau=h^{4 / 3}$. In addition, the results when $\mathrm{T}=1$ and $\mathrm{T}=2$ show the unconditional stability of the proposed algorithm.

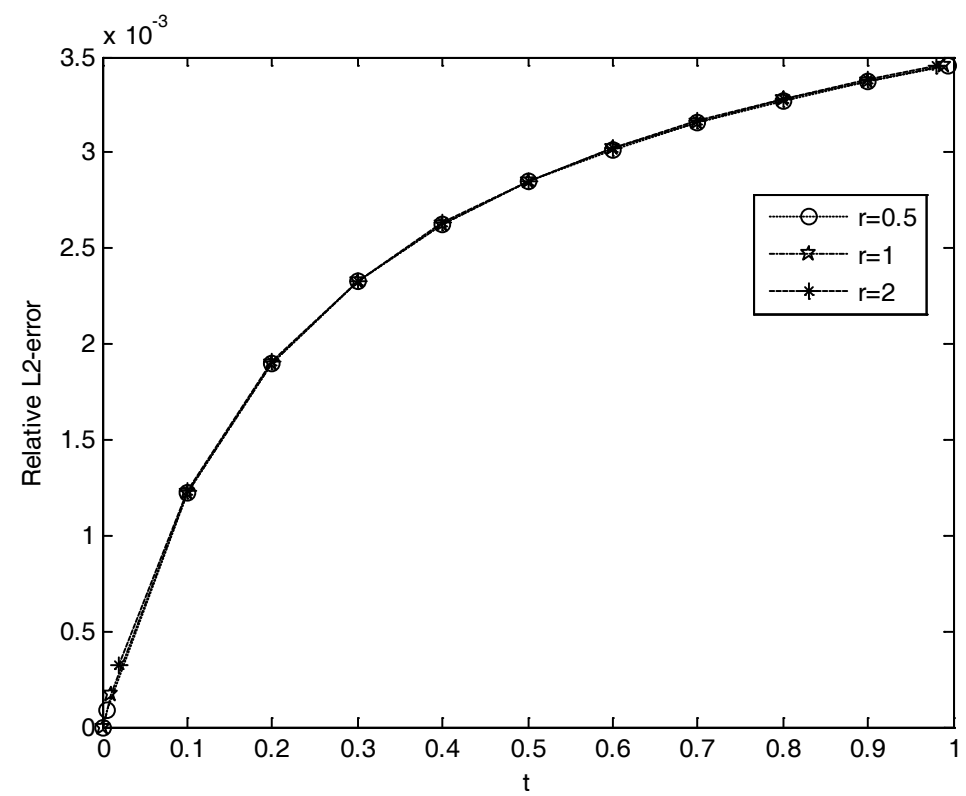

Figure 1. Relative $L^{2}$-error of Example 1 during $0 \leq t \leq 1$ under different mesh ratios for $\sigma=1$ and $M=100$.

We can also see in Figure 1 that the numerical result is unconditionally stable during the computational time period, regardless of the mesh ratio $r=\frac{\sigma \tau}{h^{2}}$.

\section{Conclusions}

The numerical solution to the non-homogenous heat equation in the unbounded domain is considered. Similar to the numerical methods of non-homogenous heat equation in the halfunbounded domain in [1] and the involved homogenous heat equation in unbounded domain in [5], we convert the original problem to an initial-boundary value problem by using two exact artificial boundary conditions. Then the finite difference scheme is obtained by the method of reduction of order. The stability and the corresponding error estimate of (19)-(23) are given in Theorem 2. A non- 
homogenous example demonstrates the unconditional stability and the accuracy of the proposed method.

\section{Acknowledgement}

This work is supported by National Natural Science Foundation of China. (11471019).

\section{References}

1. H.-D. Han, Z.-Y. Huang. A class of artificial boundary conditions for heat equation in unbounded domains, Comput. Math. Appl. 43 (2002) 889-900.

2. H.-D. Han, Z.-Y. Huang, Exact and approximating boundary conditions for the parabolic problemson unbounded domains, Comput. Math. Appl. 44 (2002) 655-666.

3. X.-N. Wu, Z.-Z. Sun, Convergence of difference scheme for heat equation in unbounded domainsusing artificial boundary conditions, Appl. Numer. Math. 50 (2004) 261-277.

4. H.-D. Han, X.-N. Wu, Artificial Boundary Method, Beijing: Tsinghua University Press/SpringerPress, 2012.

5. Q. Zheng, L. Fan, X.-Z. Li, An artificial boundary method for Burgers' equation in the unboundeddomain, CMES: Comput. Model. Eng. Sci. 100 (2014) 445-461. 